\title{
Carbon string structures: First-principles calculations of quantum conductance
}

\author{
R. T. Senger, S. Tongay, S. Dag, E. Durgun, and S. Ciraci* \\ Department of Physics, Bilkent University, 06800 Ankara, Turkey
}

(Received 24 August 2004; revised manuscript received 10 February 2005; published 9 June 2005)

\begin{abstract}
Carbon forms various nanostructures based on the monatomic chains or strings which show transport properties of fundamental and technological interest. We have carried out first-principles quantum conductance calculations using optimized structures within density functional theory. We treated finite segments of carbon monatomic chain, metal-semiconductor heterostructure, and resonant tunneling double barrier formed of C-BN chains, as well as symmetric and antisymmetric loop devices between two electrodes. We examined the effects of electrode, contact geometry, size of the device, strain, and foreign atoms adsorbed on the chain. Calculated quantum ballistic conductance of carbon chains showing even-odd disparity depending on the number of atoms and strain are of particular interest. Notably, chains consisting of an even number of carbon atoms contacted to metal electrodes display a resonant tunneling-like behavior under axial strain. The double covalent bonding of carbon atoms depicted through self-consistent charge density analysis underlies unusual transport properties.
\end{abstract}

DOI: 10.1103/PhysRevB.71.235406

PACS number(s): 73.63.-b, 73.22.-f, 73.40.Jn

\section{INTRODUCTION}

As the research on molecular electronics (or moletronics $)^{1,2}$ has been in progress for several decades, connecting those nanodevices has remained a real challenge. In an effort to develop highly conducting interconnects, intense research has focused on nanowires, ${ }^{3}$ which revealed unusual mechanical and transport properties of metallic wires. ${ }^{4-7}$ In particular, a close correlation between their atomic structure and stepwise variation of conductance has attracted interest. $^{8-10}$ Following predictions of several theoretical studies $^{11-13}$ the production of monatomic linear gold chain has been a breakthrough. ${ }^{14,15}$ More recently, multiwall and single-wall tubular wires of gold have been observed and studied. ${ }^{16-18}$ While those thin metal wires drawn between two electrodes have been useful to reveal fundamental aspects of quantum ballistic conductance, they were not reproducible, and hence were unsuitable for device applications. Carbon nanotubes ${ }^{19,20}$ with their unusual mechanical and electronic properties have been considered as a promising class of nanostructures meeting several requirements and offering new paradigms in nanoelectronics. Further to several device applications, single-wall carbon nanotubes (SWNT) are also considered as interconnects. Experimentally and theoretically it has been shown that SWNTs can be uniformly coated with Ti atoms. ${ }^{21,22}$ With their high conductivity and polarized spins at the Fermi level, coated carbon nanotubes appear to be suitable for spintronic applications. ${ }^{22}$

Another ultimate one-dimensional (1D) nanowire, namely, monatomic linear chain of carbon atoms (C-LC), attracted the interest of several researchers much earlier. ${ }^{23-29}$ Using chemical methods, production of carbon monatomic chains up to 20 atoms in length has been achieved. ${ }^{30} \mathrm{Re}-$ cently, C-LC has been observed at the center of multiwall carbon nanotubes. ${ }^{31}$

The transport properties of C-LC have attracted considerable attention owing to its ultimate size in fabricating nanodevices. Lang and Avouris ${ }^{32}$ studied the conductance variation of finite-size C-LCs having $N$ atoms $(3 \leqslant N \leqslant 7)$ in contact with two jellium electrodes. They found that the equilibrium conductance $G(N)$ varies in an oscillatory manner, with odd-numbered C-LCs having a higher conductance than even-numbered ones. This oscillatory variation of conductance was attributed to the effect of $N$ on the occupation of the degenerate $\epsilon\left(\pi_{x}\right)=\epsilon\left(\pi_{y}\right)$ levels and their modification by the coupling of jellium electrodes. The combined effect is reflected in the state density at the Fermi level of the electrode-C-LC-electrode system. The oscillatory behavior of $G(N)$ was revealed earlier also for the Na-chain structure. ${ }^{33}$ Similar arguments based on the Laughlin Kalmeyer theory ${ }^{34}$ were used in explaining the conductance through a single $\mathrm{Al}$ atom between two metal electrodes. ${ }^{35}$ Later, Lang and Avouris ${ }^{36}$ showed that there is a large transfer of charge from the electrodes to the chain, which provides doping of the chain without introducing scattering centers. Recently, Larade et al. ${ }^{37}$ reported a first-principles analysis of finite-size C-LCs with $4 \leqslant N \leqslant 7$ in contact with two crystalline Al-electrodes under external bias. Their work demonstrated that the conductance is affected by the crystalline order of electrodes, and coupling between carbon chain and $\mathrm{Al}$ electrodes is one of the major factors controlling the transport. Accordingly, whether carbon atoms were attached to the top site or hollow site on the Al electrode becomes significant as pointed out much earlier. ${ }^{35}$ Most importantly, the even-odd disparity in the number of chain atoms found by Larade et $a l .{ }^{37}$ has a trend opposite to that obtained in jellium-electrode calculations, even-numbered chains having higher conductance values than odd-numbered ones. The first-principles analysis has also revealed a negative differential resistance at finite biases owing to a shift of conduction channels relative to the states of $\mathrm{Al}$ electrodes. ${ }^{37}$

This paper presents an extensive study of the transport properties of various structures based on carbon strings using first-principles calculations of quantum conductance. Our preliminary results have been reported as a short letter. ${ }^{38}$ Here we extend our analysis to investigate the effect of electrodes, contact geometry, strain, and device-size which shows interesting even-odd disparity in the number of atoms. We also examined atomic string heterostructures and loop devices. 


\section{METHOD}

We have performed self-consistent field (SCF) total energy and electronic structure calculations using the firstprinciples pseudopotential plane wave $\operatorname{method}^{39,40}$ within density functional theory ${ }^{41}$ (DFT). All infinite and finite-size nanostructures in our study have been treated within supercell geometries. Generalized gradient approximation ${ }^{42}$ (GGA) and local density approximation ${ }^{43}$ (LDA) have been used in the calculations. The reported values are GGA results unless otherwise stated. All the atomic positions and lattice parameters of periodic structures have been optimized by minimizing the total energy, forces on atoms, and the stress on the structure. For the ultrasoft pseudopotentials ${ }^{40,44}$ used in our calculations the wave functions are represented by plane waves up to kinetic energy cutoff $|\mathbf{k}+\mathbf{G}| \cong 400 \mathrm{eV}$. The Brillouin zones of various structures have been sampled by the Monkhorst-Pack k-point sampling scheme. ${ }^{45}$ The number of k-points are determined by performing convergence tests, which yield $1 \times 1 \times 51$ mesh appropriate for the case of C-LC with a single atom per supercell. For larger supercells, the appropriate numbers of $\mathbf{k}$-points have been set by scaling according to the size of the unit cell.

The analysis of quantum ballistic conductance for infinite and finite size structures have been performed using TRANSIESTA-C, a recently developed $a b$ initio transport software based on DFT, localized basis sets, and nonequilibrium Green's function (NEGF) formalism. ${ }^{46,47}$ In TRANSIESTA-C we have employed double-zeta-parameter numerical basis sets which are usually comparable to well converged plane wave basis sets. As usual, we have used an electrode-device-electrode geometry for conductance calculations. The conductance of the device has been calculated as

$$
G(E)=\frac{2 e^{2}}{h} \operatorname{Tr}\left(\Gamma_{l} G^{r} \Gamma_{r} G^{a}\right)
$$

after iteratively solving nonequilibrium Green's function and DFT equations. ${ }^{47}$ In the above equation, $G^{r}$ and $G^{a}$ are retarded and advanced Green's functions, and $\Gamma_{l}$ and $\Gamma_{r}$ are coupling functions to the left and right electrodes, respectively. In order to match the device potential and the surface potential of the semi-infinite electrodes, the device regions are defined to contain some portions of the electrodes.

DFT is employed to describe exchange correlation and charging effects as well as the atomic structure of both the device and the electrodes in a self-consistent manner. Unlike conventional DFT simulation schemes, in order to account for the infinite and open nature of the system due to the electrodes, DFT is used in combination with NEGF formalism. The latter provides the means for representing the effects of infinite electrodes in the form of self-energy terms.

\section{RESULTS AND DISCUSSION}

Earlier, we treated the conductance of periodic, infinite $\mathrm{C}$-LC, infinite and finite helix structures $\mathrm{C}-\mathrm{HX}_{(N, p)}$ in Ref. 38 , where the mechanical and electronic structure of carbon monatomic strings are also discussed to some extent. Unlike
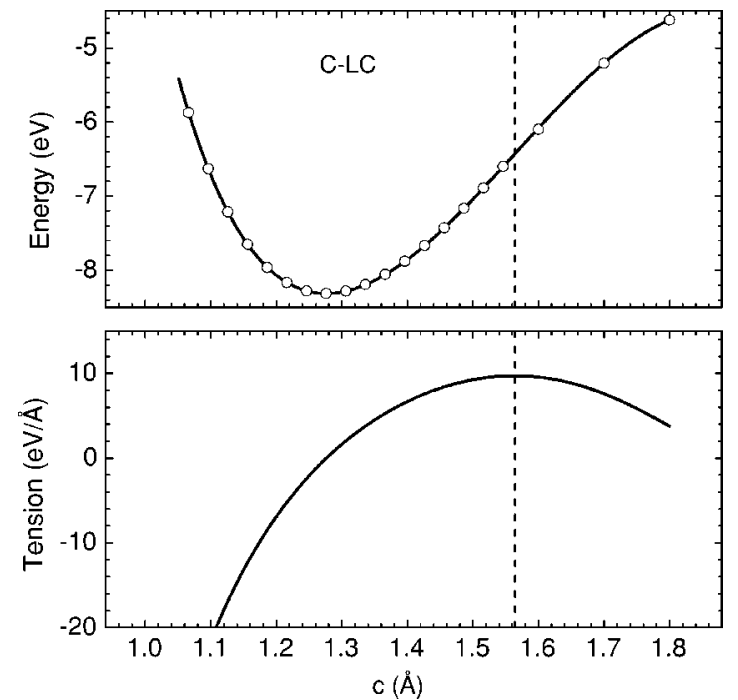

FIG. 1. Total energy per atom and tension of carbon linear chain as functions of lattice parameter. The energies are given relative to the energy of the isolated $\mathrm{C}$ atom. The energy curve is a best fit to the calculated values (circles), then the tension curve is obtained by differentiation. The vertical dashed line separates the stable and unstable domains of the chain with respect to a longitudinal deformation.

most other elements and compounds, monatomic strings of carbon do not tend to form zigzag chains. The carbon atomic chain is stiff along its axis but flexible in the transverse directions. ${ }^{38}$ In Fig. 1 the total energy and tension variations in periodic $\mathrm{C}$-LC as functions of the lattice parameter are shown. The equilibrium lattice constant is $1.27 \AA$. As the chain is stretched homogenously both the energy and the tension of the chain increase. At the point where the maximum value of the tension is reached, the velocity of sound of the longitudinal-acoustic mode is zero, and the chain breaks due to long-wavelength perturbations. ${ }^{48}$ For C-LC the slope of the tension becomes zero at $c=1.56 \AA$, which corresponds to a maximum strain value of $\epsilon=0.23$ before the chain breaks.

In the present work we are concerned with the conductance of the unstrained and strained finite segment of C-LC between different types of electrodes, the finite segment of C-LC having substitutional impurity, $(\mathrm{BN})_{3}$ barrier, $(\mathrm{BN})_{3} \mathrm{C}_{6}(\mathrm{BN})_{3}$ resonant tunneling double barrier, and asymmetric and symmetric loop devices. We will address important questions, which have not been investigated thoroughly yet and reveal the effect of size, axial strain, geometry, and also the type of electrodes on the transport properties.

In atomic scales, the detailed atomic structure of contact and the type of the electrodes have considerable effects on the conductance of the molecular conductors. We first choose to use C-LC as the metallic electrodes. The atoms in the device region are subjected to geometry optimizations in supercells, keeping the electrode-atom positions fixed. The geometry optimization has been performed also for devices under axial strain. Accordingly, interatomic distances in the device and the distance between the electrodes and the chain are determined by minimization of the total energy. We also 


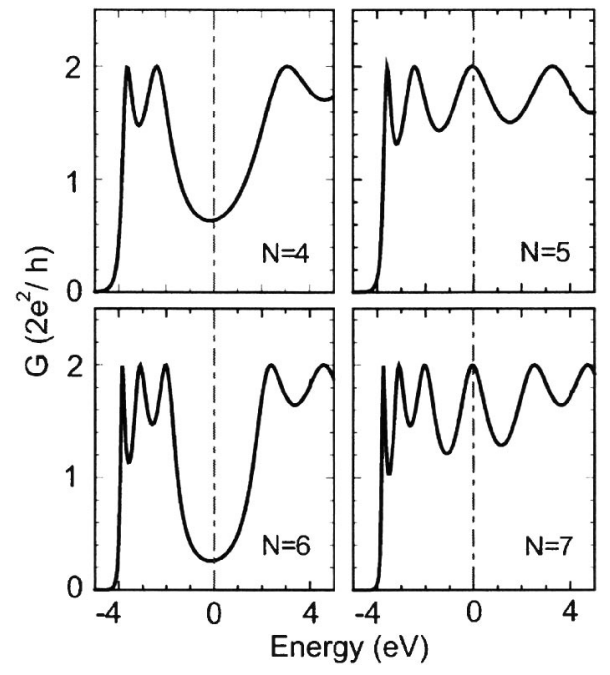

FIG. 2. Variation of calculated conductance $G(E)$ of a segment of strained C-LC placed between two semi-infinite, strain-free carbon linear chains as electrodes. Fermi levels are set to zero. Atomic positions in the segments consisting of $N=4,5,6,7$ atoms are optimized for the value of strain $\epsilon=0.15$ and resulted in nonuniform $\mathrm{C}-\mathrm{C}$ bond distances.

used $\mathrm{Al}$ electrodes having a specific crystalline orientation to reveal the effect of the electrodes on conductance properties.

\section{A. Conductance of carbon chain segments}

First, we consider the conductance of a C-LC segment under applied strain. Here the C-LC segment having $N$ carbon atoms is in contact with two electrodes taken as also semi-infinite C-LCs. By using C-LC electrodes we intend to achieve perfect contacts to the chain. Here, while semiinfinite C-LCs are kept in their ideal atomic configuration, the $\mathrm{N}$-atom segment of the chain in the device region is strained. When an axial tension $F$ is applied to an $N$-atom chain, the length $L$ of the chain increases so that $L \rightarrow L(1$ $+\epsilon$ ). We optimize the atomic positions of the elongated segment by keeping the separation of two C-LC electrodes at $L(1+\epsilon)$. The strain leads to nonuniform bond lengths in the device region. For example, in a six-atom C-LC under a strain of $\epsilon=0.15$ the sequence of equilibrium bond lengths becomes $1.56,1.31,1.59,1.30,1.59,1.31,1.56 \AA$ after relaxation. The lengths of the bonds between the terminal atoms of the chain and the electrodes are equal to $1.56 \AA$. The resulting conductance spectrum of these strained chains displays oscillatory behavior with $E$. Figure 2 presents $G(E)$ of a finite C-LC device containing $N=4-7$ atoms subjected a strain of $\epsilon=0.15$. The character of oscillations is dramatically different depending on $N$ being odd or even. If $N$ is odd, the chain always has a peak conductance value of $2 G_{0}$ at the Fermi level, whereas for even $N, G\left(E_{F}\right)$ corresponds to a minimum of oscillations which drops considerably with increasing $\epsilon$. This salient feature is related to the alignment of the molecular energy levels of the device region with the electrochemical potential of the electrodes. It turns out that $E_{F}$ coincides with the HOMO for odd-numbered chains, and with the center of the HOMO-LUMO gap for even-

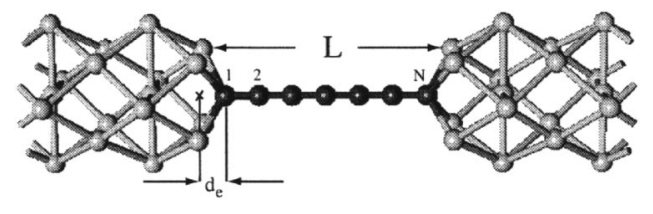

FIG. 3. The electrode-device-electrode model used for the calculation of conductance of finite C-LC consisting of $N=4,5,6,7$ atoms. Electrodes are taken as $\mathrm{Al}(001)$ bars made by consecutive squares and centered squares (rotated by $45^{\circ}$ ) representing (001) planes of an $\mathrm{Al}$ fcc crystal perpendicular to the chain axis. $L$ is the spacing between two electrode surfaces, $d_{e}$ is the distance between the electrode surfaces and the terminal atoms of the C-LC segment.

numbered ones. When geometrical optimization of the chain is omitted and the strain is assumed to be uniform the conductance oscillations are still present but their amplitudes are smaller. ${ }^{38}$ The observation that even-numbered, strained carbon chains have lower conductance values is compatible with the findings of Lang and Avouris for unstrained chains. ${ }^{32}$ They reported $G \simeq 1.8 G_{0}$ for carbon chains with $N=5$ and 7 in between jellium metal electrodes. In our model, the use of C-LC electrodes naturally corresponds to a better or even perfect contact that leads to $G=2 G_{0}$. With our choice of electrodes, unlike the results in Ref. 32, the difference of $N$ being odd or even in the calculated conductance of unstrained $(\epsilon=0)$ carbon chain does not manifest itself, because for all $N$ the configuration is the same. Therefore the odd-even $N$ disparity we find in the conductance of carbon chains is a pure strain-induced effect.

To study the effect of electrodes on the strain-free and strained carbon chains we also consider another system where a finite size (or a segment of) C-LC is in contact with two metal electrodes. As metal electrodes, we consider $\mathrm{Al}$ bars consisting of (001) planes, where [001] crystalline direction is taken parallel to the chain axis. The atomic positions of atoms in the $\mathrm{Al}$ electrodes have been optimized in the infinite wire configuration. Once the $N$-atom chains are connected to the hollow sites of the $\mathrm{Al}$ electrodes, the atomic positions of $\mathrm{C}$ atoms are optimized by minimizing the total energy and stress while keeping the distance $L$ between the two $\mathrm{Al}$ electrodes fixed. In that optimization step, $\mathrm{C}$ atoms are allowed to move but $\mathrm{Al}$ electrodes are assumed to be rigid. It should be pointed out that the assumption of rigid electrodes is an approximation, in particular for strained systems. However, we consider these as model systems and our emphasis is on the response of carbon atomic chains to external strains. In principle, the part of the electrodes in the device region should also be allowed to relax, but deformations in the electrodes would prohibit one to attribute the conductance modifications to the carbon chain alone. In our electrode-device-electrode configuration, each device is a segment of C-LC consisting of $N$ atoms $(N=4,5,6,7)$ as shown in Fig. 3. It is similar to the model considered in Ref. 37 , except that in the present case chain-electrode distances, $d_{e}$, and all $\mathrm{C}-\mathrm{C}$ bond lengths are optimized.

The optimized (equilibrium) carbon positions at zero strain exhibit interesting features worth emphasizing: First, the $\mathrm{C}-\mathrm{C}$ bond lengths are not uniform. For $N=4, d_{e}$ $=0.84 \AA$ (or $\left.d_{\mathrm{Al}-\mathrm{C}}=2.2 \AA\right)$; and the sequence of equilibrium 


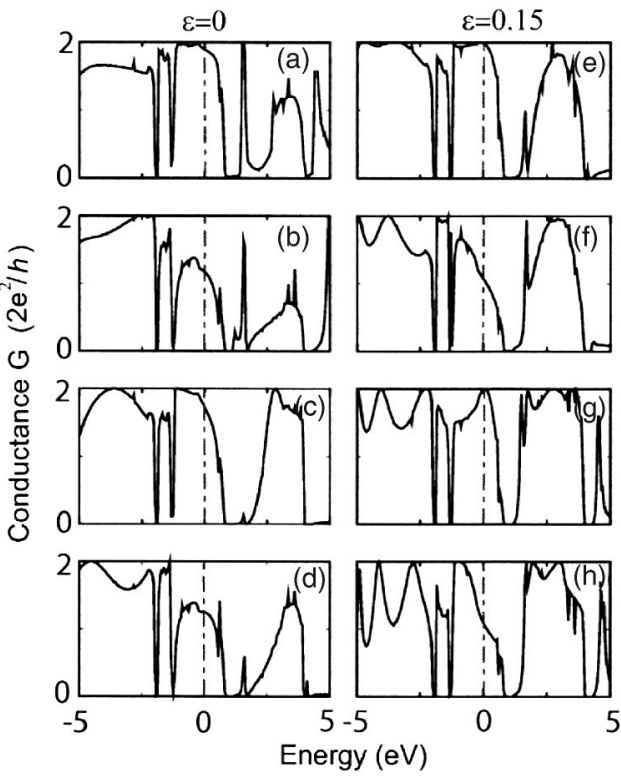

FIG. 4. (a)-(d) Variation of conductance vs energy $E, G(E)$, calculated for relaxed (strain free) C-LC segments consisting of $N$ $=4,5,6,7$ atoms, respectively, in between $\mathrm{Al}$ electrodes as described in Fig. 3. The Fermi energy $E_{F}$ is set to zero. (e)-(h) Same for the C-LC segments under a strain $\epsilon=0.15$.

$\mathrm{C}-\mathrm{C}$ bond lengths starting from the first carbon atom in Fig. 3 are $1.34,1.25$, and $1.34 \AA$. Whereas for $N=5, d_{e}$ $=0.92 \AA$ (or $d_{\mathrm{Al}-\mathrm{C}}=2.3 \AA$ ); and the sequence of four equilibrium $\mathrm{C}-\mathrm{C}$ bond lengths is $1.34,1.26,1.26$, and $1.34 \AA$. Considering $N=6$ and 7 , the equilibrium distance from the end atom of the chain to the last atomic plane of electrode changes in the range $0.84 \leqslant d_{e} \leqslant 0.95 \AA$; as for the $\mathrm{C}-\mathrm{C}$ bond lengths, they are nonuniform with specific symmetry and vary in the range $1.25 \leqslant d_{i, i+1} \leqslant 1.34 \AA$.

The variation of calculated equilibrium conductance of the system described in Fig. 3 with a finite segment of C-LC comprising a different number of atoms $(N=4-7)$ under $\epsilon$ $=0$ is shown in Figs. 4(a)-4(d) in the energy range $-5 \mathrm{eV}$ $\leqslant E \leqslant 5 \mathrm{eV}$. The conductance spectrum is derived from the electronic structure of two electrodes coupled to the $\mathrm{N}$-carbon atom chain for $\mathrm{Al}$ electrodes. The electronic energy level structure of the chain strongly depends on $N$ and $\mathrm{C}-\mathrm{C}$ bond lengths, $d_{i, i+1}$, and it shifts and broadens depending on the strength of coupling with metal electrodes. Eventually, the resulting transmission coefficient and $G(E)$ incorporate all these effects self-consistently.

Figure 5 shows the dependence of the equilibrium conductance values on the number of carbon atoms present in the chain (zero strain). The value of $G\left(E=E_{F}\right)$ varies between $\sim 1.8$ and $1.2 G_{0}$ for optimized C-LC segments with $\mathrm{Al}$ electrodes and under $\epsilon=0$. As far as the even-odd disparity is concerned, the trend depicted in Fig. 5 agrees with the trend reported by Larade et al., ${ }^{37}$ but disagrees with the variation obtained from jellium calculations by Lang and Avouris. ${ }^{32}$

In order to see the effect of strain, we studied the same system described in Fig. 3, for $\epsilon=0.15$, so that the spacing between $\mathrm{Al}$ electrodes has become $L(1+\epsilon)$. Having increased

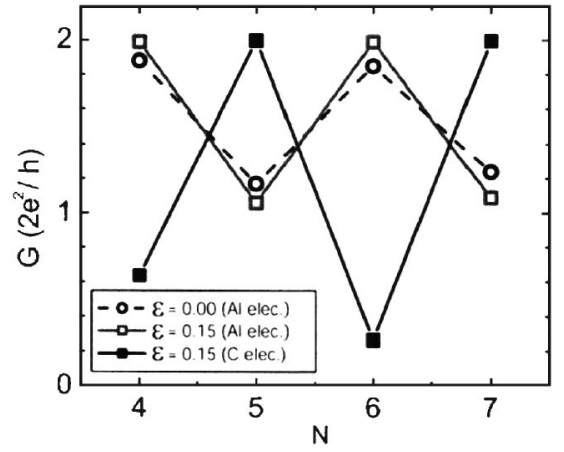

FIG. 5. Variation of calculated equilibrium conductance, $G(E$ $=E_{F}$ ), of C-LC segments for $\epsilon=0$ and 0.15 , as a function of $N$, the number of atoms in the segment. The atomic positions in the segment and the distance to $\mathrm{Al}$ electrodes are optimized for each case. The $N$-atom C-LC segment is in contact with two $\mathrm{Al}$ electrodes as described in Fig. 3. The variation of $G\left(E=E_{F}\right)$ of strained C-LC segments between two C-LC electrodes as a function of $N$ is shown for the sake of comparison.

the spacing $L$, the positions of $N$ carbon atoms in the segment have been determined again by optimization. To exclude the effect of the site where the chain makes contact, we constrained the chain to be connected to the hollow-site as in the case of $\epsilon=0$. Without this constraint, the chain under strain favored the contact at the axial site, namely, a bridge site above two adjacent $\mathrm{Al}-\mathrm{Al}$ bonds. Such a constrained structure optimization is of importance, since the bond distances of the strained finite C-LC segment are not uniform, instead they exhibit variation depending on $N$. Especially, the distance from the first and $N$ th carbon atoms to the electrodes, $d_{e}$, is crucial for the coupling between C-LC and Al electrodes. An earlier study by Larade et al. ${ }^{37}$ examined the effect of $d_{e}$ on the transport properties of a C-LC segment by varying the value of $d_{e}$.

The present study first determines what the required value of $d_{e}$ should be. Then the corresponding conductance is calculated. The conductance of a segment of C-LC between two electrodes depends on the details of its electronic level structure relative to the Fermi level of the metal electrodes. The strength of the coupling to electrodes also induces changes on the detailed electronic structure. The values of $d_{e}$ and all bond lengths, $d_{i, i+1}$, optimized under a given strain of $\epsilon$ $=0.15$ reveal interesting features. As in the case of $\epsilon=0$, the bond lengths remain to be nonuniform under strain. However, the dramatic changes occur in the values of $d_{e}$. For $N$ $=4,5,6,7$, the optimized $d_{e}\left(\right.$ or $\left.d_{\mathrm{Al}-\mathrm{C}}\right)$ values at the contact are calculated to be 1.23 (2.95), 1.37 (3.00), 1.42 (3.03), and 1.54 (3.12) $\AA$, respectively. We explain this situation by a carbon-Al-electrode coupling which is relatively weaker than the strong $\mathrm{C}-\mathrm{C}$ bonds. It also shows that, under higher strains, the chain would break at the contact points to the electrodes rather than at a $\mathrm{C}-\mathrm{C}$ bond.

In Fig. 4 we also show the calculated $G(E)$ curves for C-LC segments under $\epsilon=0.15$ strain. The values of $G(E)$ for $E=E_{F}$ are plotted in Fig. 5 to show how the equilibrium conductance depends on $N$ under strain. We see that the even-odd $N$ disparity in conductance continues to exist under $\epsilon=0.15$. An interesting issue one observes in Fig. 5 is that $G$ 


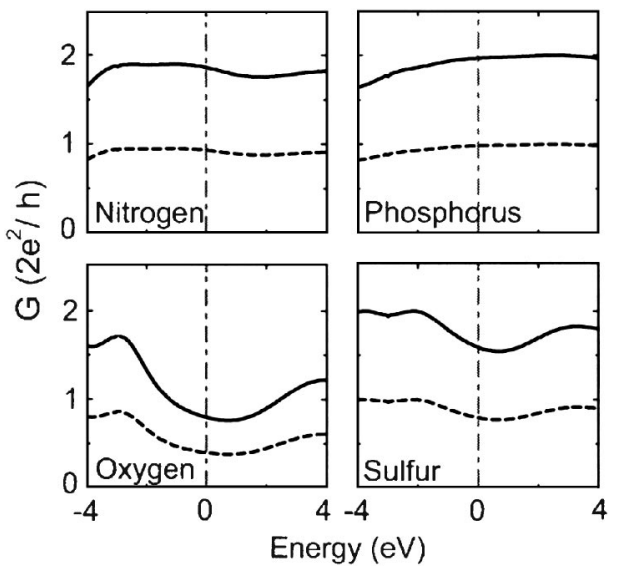

FIG. 6. Variation of calculated conductance $G(E)$ of a segment of C-LC having a single $(\mathrm{N}, \mathrm{P}, \mathrm{O}, \mathrm{S})$ substitutional impurity. The dashed lines delineate the conductance of each of the two equivalent eigenchannels of transmission. The variation of the total conductance $G$ is shown by the solid lines.

increases for even $N$ in spite of the fact that the contact to the electrodes gets weaker (or stated differently $d_{e}$ becomes larger under strain). For example, for $N=4$ and 6 the conductance becomes very close to the perfect value, i.e., $G(E$ $\left.=E_{F}\right) \rightarrow 2 G_{0}$. This means that the effect of scattering from the contact is totally eliminated. This situation suggests a resonant tunneling-like behavior between metal electrodes and the carbon chain, even though the present system corresponds to a metal-metal contact, and is not in the tunneling regime. The nontrivial variation of equilibrium conductance under applied strain is due to the detailed effects of charging and broadening in the molecular energy levels of the chain when coupled to the electrodes. Variations in $d_{e}$ change the degree of the coupling, and the relative weights of competing effects of charging and broadening. In Fig. 5 we also plot the variation of the conductance at the Fermi level, $G\left(E=E_{F}\right)$ of the $\epsilon=0.15$ strained C-LC segment placed between two C-LC electrodes as a function of $N$. We see a reverse trend compared to the case with $\mathrm{Al}$ electrodes. The situation depicted in Fig. 5 indicates that $G\left(E=E_{F}\right)$ is strongly dependent on the type of electrodes and the contact geometry between the segments and the electrodes.

\section{B. Effect of substitutional doping}

We considered seven-atom C-LC segments containing N, $\mathrm{P}, \mathrm{O}$, or $\mathrm{S}$ as a substitutional impurity atom. As shown in Fig. 6 the conductance $G(E)$ of a segment of C-LC including a substitutional impurity is strongly affected. We again used rigid C-LC electrodes. During the structural optimization of the device region, the substitution of impurity atoms alters a few neighboring $\mathrm{C}-\mathrm{C}$ bond lengths. With the particular $\mathrm{C}$-LC electrodes the problem corresponds to calculating the conductance of infinite C-LC with a single impurity atom at the origin. In Landauer-Büttiker formalism, the conductance of a device is defined through the transmission matrix, diagonalization of which leads to a set of eigenchannels. The total transmission is obtained as the sum of the contributions of

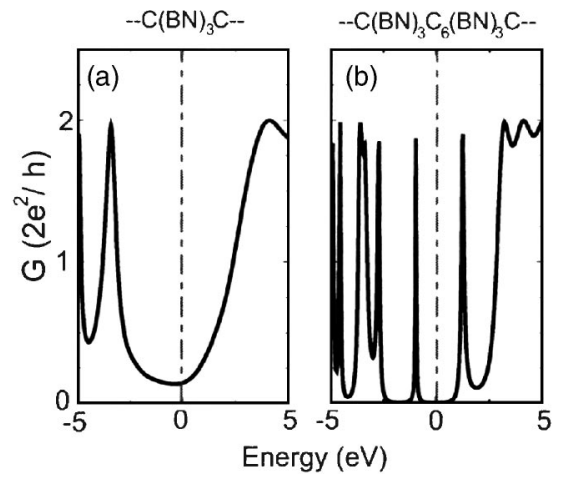

FIG. 7. (a) The conductance $G(E)$ of a $(\mathrm{BN})_{3}$ barrier in between two C-LC electrodes. (b) $G(E)$ of a double barrier structure, $(\mathrm{BN})_{3} \mathrm{C}_{6}(\mathrm{BN})_{3} . E_{F}$ is set to zero.

the individual channels. In Fig. 6, in all cases of substitutionally doped C-LC, there are two eigenchannels that make identical contributions to the total conductance.

While the decrease in conductance at $E_{F}$ is minor for N and $\mathrm{P}$ impurity, $\mathrm{S}$ causes a significant decrease with a characteristic pattern. The effect of substitutional $\mathrm{O}$ impurity is even more dramatic causing a substantial fall of conductance at the Fermi level. It should be noted that the variation of conductance $G(E)$ is highly sensitive to the atomic positions in the segment and on the distance to the electrodes. The present variation of $G(E)$ for P impurity at $E=E_{F}$ is slightly different from that in Ref. 38 owing to an improved structure optimization in the present calculations. Present results suggest that C-LC can be easily doped and its conductance can be modified.

\section{Conductance of various devices}

The binary compound BN can form a stable linear structure and displays properties very similar to that of C-LC, except that it is a wide band gap insulator. The band gap of $\mathrm{BN}$ has been calculated to be $5 \mathrm{eV}$. Normally, a C-LC$\mathrm{BN}-\mathrm{LC}$ junction is a metal-semiconductor heterostructure. A periodic arrangement of $\mathrm{C}_{N}(\mathrm{BN})_{M}$ forms a superlattice; it is a semiconductor with a band gap depending strongly on $N$ and $M$. Here we consider two different devices composed from a $\mathrm{C}-\mathrm{LC}$ and BN-LC heterostructure. The first device is a short $(\mathrm{BN})_{3}$ segment placed in between two C-LC electrodes. In the case of a short segment, the BN-LC has HOMO and LUMO states separated by a wide gap. The variation of equilibrium conductance $G(E)$ is illustrated in Fig. 7(a). A HOMO-LUMO gap of $\sim 5 \mathrm{eV}$ is present around the Fermi level. The double barrier structure $(\mathrm{BN})_{3} \mathrm{C}_{6}(\mathrm{BN})_{3}$ that connected two C-LC electrodes from left and right can be envisaged as a resonant tunneling double barrier structure. The conductance $G(E)$ in equilibrium in Fig. 7(b) reflects the electronic structure of the C-BN string heterostructure.

Ring structures of carbon are also present, and they are studied in several recent works. ${ }^{24-28}$ We have considered the $\mathrm{C}-\mathrm{R}_{14}$ ring structure to form a loop device. The circular ring connected to short C-LC segments from both sides has been optimized. Here we consider a symmetric and an asymmetric 


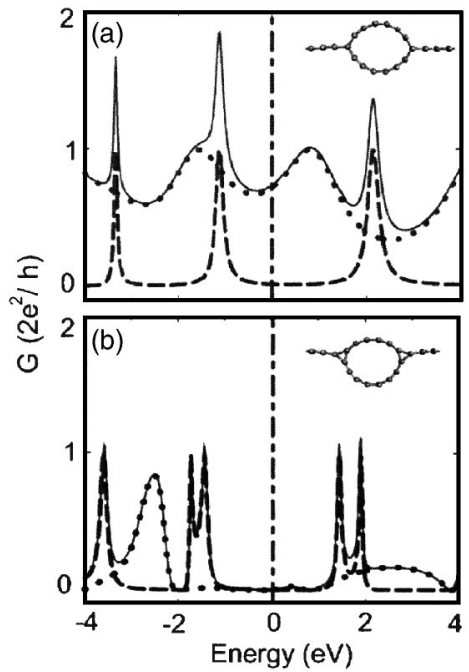

FIG. 8. The conductance $G(E)$ of a loop device for (a) symmetric and (b) asymmetric connection with C-LC electrodes. The two eigenchannels and the total conductance are shown by dashed, dotted, and solid curves, respectively.

loop device and calculate the variation of their conductance. The atomic structure of these two devices is described in Fig. 8 as an inset. After the structural relaxation the circular shape of the ring is deformed in both devices. Carbon atoms of the ring connecting to the C-LC segments from both sides form planar $s p^{2}$ bonds. As a result, the circular shape becomes oval. Figure 8 illustrates calculated total $G(E)$ together with the contributions of two eigenchannels. In the case of a symmetric loop the conductance is smaller than $G_{0}$ at $E_{F}$, but we see well-defined peaks of $G(E)$ at certain energies. While one of the eigenchannels has a smoothly oscillating pattern, the other channel's contribution occurs as sharp peaks of $\sim G_{0}$ at certain energies. As a result, the symmetric loop device is metallic. The situation is dramatically different for the asymmetric device. Here the contribution of both eigenchannels vanishes for a wide range of energies around $E_{F}$, but the device conductance has some sharp peaks at some resonant values of energy.

\section{CONCLUSIONS}

In this work we presented an $a b$ initio study of transport properties of various structures and devices based on carbon and carbon-boron-nitride heterostructure strings. These structures show unusual mechanical, electronic, and transport properties. We examined the effect of the electrodes and contact geometry, the effect of size, geometry, and strain of the string structures between electrodes.

As far as technological applications of interconnects or nanodevices are concerned, the transport properties of carbon string structures are of particular interest. We carried out an extensive analysis of the transport properties of segments of strained carbon linear chains including a different number of carbon atoms placed between different types of electrodes. In particular, the conductance calculations for a chain segment of $N$ carbon atoms contacted to $\mathrm{Al}$ electrodes from both ends revealed interesting odd-even $N$ disparity and resonant tunneling-like behavior under strain.

\section{ACKNOWLEDGMENT}

S.C. acknowledges the partial support by TÜBA (The Turkish Academy of Sciences).
*Electronic address: ciraci@fen.bilkent.edu.tr

${ }^{1}$ A. Aviran and M. A. Ratner, Chem. Phys. Lett. 29, 277 (1974).

${ }^{2}$ S. Roth and C. Joachim, Atomic and Molecular Wires (Kluwer, Dordrecht, 1997); Molecular Electronics, edited by J. Jortner and M. A. Ratner (Blackwell, Oxford, U.K., 1997).

${ }^{3}$ C. Joachim et al., Nature (London) 408, 541 (2000).

${ }^{4}$ N. Agraït, J. G. Rodrigo, and S. Vieira, Phys. Rev. B 47, R12345 (1993)

${ }^{5}$ J. I. Pascual, J. Mendez, J. Gomez-Herrero, A. M. Baro, N. Garcia, and V. T. Binh, Phys. Rev. Lett. 71, 1852 (1993).

${ }^{6}$ J. M. Krans, C. J. Müller, I. K. Yanson, T. C. M. Govaert, R. Hesper, and J. M. van Ruitenbeek, Phys. Rev. B 48, R14721 (1993).

${ }^{7}$ For a recent review of the subject see, S. Ciraci, A. Buldum, and I. P. Batra, J. Phys.: Condens. Matter 13, R537 (2001).

${ }^{8}$ S. Ciraci and E. Tekman, Phys. Rev. B 40, R11969 (1989).

${ }^{9}$ N. Agraït, G. Rubio, and S. Vieira, Phys. Rev. Lett. 74, 3995 (1995).

${ }^{10}$ A. Stalder and U. Dürig, Appl. Phys. Lett. 68, 637 (1996).

${ }^{11}$ H. Mehrez and S. Ciraci, Phys. Rev. B 56, 12632 (1997).

${ }^{12}$ O. Gulseren, F. Ercolessi, and E. Tosatti, Phys. Rev. Lett. 80, 3775 (1998).
${ }^{13}$ M. R. Sorensen, M. Brandbyge, and K. W. Jacobsen, Phys. Rev. B 57, 3283 (1998).

${ }^{14}$ H. Ohnishi, Y. Kondo, and K. Takayanagi, Nature (London) 395, 780 (1998).

${ }^{15}$ A. I. Yanson, G. R. Bolliger, H. E. van der Brom, N. Agraït, and J. M. van Ruitenbeek, Nature (London) 395, 783 (1998).

${ }^{16}$ Y. Kondo and K. Takayanagi, Science 289, 606 (2000).

${ }^{17}$ Y. Oshima, A. Onga, and K. Takayanagi, Phys. Rev. Lett. 91, 205503 (2003)

${ }^{18}$ R. T. Senger, S. Dag, and S. Ciraci, Phys. Rev. Lett. 93, 196807 (2004).

${ }^{19}$ S. Iijima, Nature (London) 354, 56 (1991); S. Iijima, T. Ichihashi, and Y. Ando, ibid. 356, 776 (1992).

${ }^{20}$ M. S. Dresselhaus, G. Dresselhaus, and P. C. Eklund, Science of Fullerenes and Carbon Nanotubes (Academic, San Diego, 1996).

${ }^{21}$ H. Dai, E. W. Wong, Y. Z. Lu, S. Fan, and C. M. Lieber, Nature (London) 375, 769 (1995); Y. Zhang and H. Dai, Appl. Phys. Lett. 77, 3015 (2000); Y. Zhang, N. W. Franklin, R. J. Chen, and H. Dai, Chem. Phys. Lett. 331, 35 (2000).

${ }^{22}$ S. Dag, E. Durgun, and S. Ciraci, Phys. Rev. B 69, 121407(R) (2004). 
${ }^{23}$ A. Karpfen, J. Phys. C 12, 3227 (1979).

${ }^{24}$ R. O. Jones and G. Seifert, Phys. Rev. Lett. 79, 443 (1997).

${ }^{25}$ T. Wakabayashi, M. Kohno, Y. Achiba, H. Shiromaru, T. Momose, T. Shida, K. Naemura, and Y. Tobe, J. Chem. Phys. 107, 4783 (1997).

${ }^{26}$ E. J. Bylaska, J. H. Weare, and R. Kawai, Phys. Rev. B 58, R7488 (1998).

${ }^{27}$ M. Saito and Y. Okamoto, Phys. Rev. B 60, 8939 (1999).

${ }^{28}$ T. Torelli and L. Mitas, Phys. Rev. Lett. 85, 1702 (2000).

${ }^{29}$ A. Abdurahman, A. Shukla, and M. Dolg, Phys. Rev. B 65, 115106 (2002).

${ }^{30} \mathrm{G}$. Roth and H. Fischer, Organometallics 15, 5766 (1996).

${ }^{31}$ X. Zhao, Y. Ando, Y. Liu, M. Jinno, and T. Suzuki, Phys. Rev. Lett. 90, 187401 (2003).

${ }^{32}$ N. D. Lang and Ph. Avouris, Phys. Rev. Lett. 81, 3515 (1998); 84, 358 (2000).

${ }^{33}$ N. D. Lang, Phys. Rev. B 52, 5335 (1995); Phys. Rev. Lett. 79, 1357 (1997).

${ }^{34}$ V. Kalmeyer and R. B. Laughlin, Phys. Rev. B 35, 9805 (1987).

${ }^{35}$ H. Mehrez, S. Ciraci, A. Buldum, and I. P. Batra, Phys. Rev. B 55, R1981 (1997).

${ }^{36}$ N. D. Lang and Ph. Avouris, Phys. Rev. Lett. 84, 358 (2000).
${ }^{37}$ B. Larade, J. Taylor, H. Mehrez, and H. Guo, Phys. Rev. B 64, 075420 (2001).

${ }^{38}$ S. Tongay, R. T. Senger, S. Dag, and S. Ciraci, Phys. Rev. Lett. 93, 136404 (2004).

${ }^{39}$ M. C. Payne, M. P. Teter, D. C. Allen, T. A. Arias, and J. D. Joannopoulos, Rev. Mod. Phys. 64, 1045 (1992).

${ }^{40}$ Numerical calculations have been carried out by using VASP software package: G. Kresse and J. Hafner, Phys. Rev. B 47, R558 (1993); G. Kresse and J. Furthmüller, ibid. 54, 11169 (1996).

${ }^{41}$ W. Kohn and L. J. Sham, Phys. Rev. 140, A1133 (1965).

${ }^{42}$ J. P. Perdew, K. Burke, and M. Ernzerhof, Phys. Rev. Lett. 77, 3865 (1996).

${ }^{43}$ J. P. Perdew and A. Zunger, Phys. Rev. B 23, 5048 (1981).

${ }^{44}$ D. Vanderbilt, Phys. Rev. B 41, R7892 (1990).

${ }^{45}$ H. J. Monkhorst and J. D. Pack, Phys. Rev. B 13, 5188 (1976).

${ }^{46}$ L. V. Keldysh, Sov. Phys. JETP 20, 1018 (1965).

${ }^{47}$ The methodology of the TRANSIESTA-C software package is described in: M. Brandbyge, J.-L. Mozos, P. Ordejón, J. Taylor, and K. Stokbro, Phys. Rev. B 65, 165401 (2002). The software was provided by Atomistix Corp.

${ }^{48}$ F. J. Ribeiro and M. L. Cohen, Phys. Rev. B 68, 035423 (2003). 